\title{
Políticas de saúde para o controle do tabagismo na América Latina e Caribe: uma revisão integrativa
}

\author{
Health policies for tobacco control \\ in Latin America and the Caribbean: an integrative review
}

Luís Gustavo da Silva Fagundes ${ }^{1}$

Marlene das Graças Martins ${ }^{2}$

Evandro Monteiro de Sá Magalhães ${ }^{1}$

Patrícia de Cássia Ruela Palmiéri ${ }^{2}$

Sinézio Inácio da Silva Júnior ${ }^{3}$

${ }^{1}$ Departamento de Biociências, Universidade Federal de Alfenas. R. Gabriel Monteiro da Silva 714, Centro. 37.130-000 Alfenas MG Brasil.

gufagundes@yahoo.com.br ${ }^{2}$ Departamento de

Enfermagem, Universidade Federal de Alfenas.

${ }^{3}$ Departamento de Ciências Biológicas, Universidade

Federal de Alfenas.
Abstract The habit of smoking is an increasing concern for governments, health authorities and society as a whole, as it is a legal drug, which is addictive and leads to serious damage to health. Cigarette smoking causes about 5.4 million deaths/ year worldwide and is the second major risk factor for deaths. An attempt is made to identify how policies can contribute to the eradication of smoking in Latin America and the Caribbean. An integrative review was conducted at BIREME using LILACS, and 16 articles were selected using inclusion and exclusion criteria. The articles were compared and grouped according to similarity of content and divided into 4 groups: smoke-free spaces, Framework-Convention, local Brazilian policies and others. Little data about smoking in Latin America and the Caribbean is available, mainly with respect to the evaluation of policies deployed. Policies for smoke-free spaces were emphasized (5 articles), as well as the Framework-Convention on tobacco control (4 articles) and articles that portray Brazilian policies for tackling smoking (3 articles).

Key words Health policy, Tobacco, Smoking, Tobacco smoke pollution, Control
Resumo O hábito de fumar preocupa cada vez mais, os governos, as autoridades de saúde e a sociedade como um todo, por ser uma droga lícita, causar dependência e levar a sérios danos à saúde. O tabagismo causa cerca de 5,4 milhões óbito/ano no mundo, sendo o segundo fator de risco para óbitos. Buscamos identificar como as politicas podem contribuir para a cessação do tabagismo no âmbito da América Latina e Caribe. Realizou-se uma revisão integrativa, na Bireme, utilizandose o Lilacs. Obteve-se 16 artigos, selecionados pelos critérios de inclusão e exclusão. Foram comparados e agrupados por similaridade de conteúdos e divididos em 04 grupos: espaços livres de fumo, convenção quadro, políticas locais brasileiras e outros. Poucos dados sobre o tabagismo na América Latina e Caribe estão disponíveis, principalmente no que tange a avaliação das politicas implantadas. Destacou-se às políticas de espaço livres de fumo (05 artigos), a Convenção Quadro de Controle do Tabagismo (04 artigos) e artigos que retratam politicas brasileiras de enfretamento ao tabagismo (03 artigos).

Palavras-chave Politica de saúde, Tabaco, Tabagismo, Poluição por fumaça de tabaco, Controle 


\section{Introdução}

O hábito de fumar tem preocupado cada vez mais os governos, as autoridades de saúde e a sociedade como um todo, por ser uma droga lícita, causar dependência e causar sérios danos à saúde, com consequências sociais, econômicas e sanitárias. Além dos malefícios causados à saúde do próprio fumante, a fumaça exalada pelo consumo dos derivados do tabaco também é prejudicial à saúde da coletividade e ao meio ambiente ${ }^{1}$.É considerado uma epidemia mundial, sendo um grave problema de saúde pública. Apresenta-se como uma doença complexa, exigindo uma abordagem também complexa para lidar com ele ${ }^{2}$.

Dados da Organização Mundial de Saúde ${ }^{3}$ estimam que o tabagismo seja responsável por cerca de 5,4 milhões de óbitos/ano em todo o mundo, sendo, portanto, o segundo fator de risco para óbitos, só perdendo para hipertensão arterial sistêmica ${ }^{4}$. Os fumantes vivem, em média 10 anos menos do que os não fumantes e com pior qualidade de vida. Apesar dos esclarecimentos sobre seus malefícios, ainda 1,3 bilhões de pessoas fumam em todo o mundo. As principais razões para explicar a elevada prevalência de mortes em fumantes decorrem das doenças relacionadas ao tabaco serem crônicas, do seu baixo preço em muitos países, e dificuldades para deixar de fumar, pois a maioria são dependentes da nicotina, além de aspectos socioeconômicos e culturais associados ${ }^{5}$.

Ao se analisar como se dá a criação de políticas de controle do tabagismo queremos, com isto, identificar como tais políticas podem contribuir para a cessação do tabagismo, bem como se dão os processos de implantação destas políticas. Entendemos políticas como decisões de caráter geral, destinadas a tornar públicas as intenções de atuação do governo, através de programas e projetos, permitindo o acesso da população em geral, e dos formadores de opinião em particular, à discussão, à implementação e à avaliação das políticas ${ }^{6}$. A política de saúde faz parte de inter-relações com fatores que transcendem o universo da atenção à saúde, posto que seus resultados dependem também de determinantes sociais, econômicos e políticos. Assim, como qualquer outro processo decisório, a política de saúde é fruto de um complexo jogo de negociações e confrontações entre a burocracia estatal, profissionais de saúde, sindicatos, partidos políticos, grupos de interesse e associações da sociedade civil. Portanto, não só os governos se envolvem na formulação de políticas. Outros atores estão envolvidos na arena política, tais como, os grupos de interesse e os movimentos sociais, cada qual com seu papel, tendo maior ou menor destaque a depender do tipo de política formulada e das coalizões que integram o governo, tornando a atividade de governar e de formular políticas mais complexa?

Diante de todos os malefícios causados pelo hábito de fumar, e pela alta carga de mortes e de redução da qualidade de vida dos usuários, várias ações vêm sendo elencadas por todos os países, a fim de reduzir ou cessar com este hábito. Como propostas que se consolidam de forma mais ampla, abrangendo amplos pontos de combate ao tabagismo, a saúde pública mundial conseguiu um avanço muito grande, em fevereiro de 2005, que foi o primeiro tratado internacional que as Nações Unidas (ONU) aprovaram para organizar o combate a uma das indústrias mais lucrativas e poderosas do capitalismo contemporâneo. Denominado Convenção-Quadro para o Controle do Tabaco, surge devido ao crescimento do mercado global dos produtos do tabaco, caracterizado por inúmeras maneiras de expansão do mercado ilegal, envolvendo países em desenvolvimento e desenvolvidos, fato que permitiu a agilidade global de grandes companhias de tabaco, com países abertos às suas demandas e pessoas predispostas ao consumo de seus produtos. Essa convenção se caracterizou por propagar o tabagismo como um problema de saúde pública, com demanda ampla e necessidade de cooperação internacional para uma resposta eficaz, apropriada e integral, tendo em vista as devastadoras consequências sanitárias, sociais, econômicas e ambientais geradas pela exposição à fumaça do tabaco, em todo o mundo; além de impôr ônus às famílias, aos pobres e aos sistemas nacionais de saúde ${ }^{8}$. Este estudo, portanto, visa enfocar as políticas de saúde criadas para controle do tabagismo, no âmbito da América Latina e Caribe.

\section{Epidemiologia do tabagismo}

Atualmente, o tabagismo é considerado pela Organização Mundial de Saúde (OMS) como a principal causa evitável de morte em todo o mundo. Somente no ano de 2008 aproximadamente 5 milhões de indivíduos morreram devido ao cigarro. A estimativa é de que haja 8 milhões de óbitos relacionados ao tabagismo até o ano de 2030 , sendo que aproximadamente $70 \%$ acontecerão nos países em desenvolvimento ${ }^{3}$. $\mathrm{O}$ panorama mundial é bastante diverso entre os diferentes países e considera-se que a América Latina 
encontra-se no estagio III da curva epidêmica do uso do tabaco, caracterizado pela redução na prevalência entre os homens e discreto aumento ou estabilização na prevalência entre as mulheres. Esse estágio apresenta ainda grande aumento na mortalidade entre homens, decorrente de doenças relacionadas ao tabaco e, nas mulheres, essa mortalidade ainda é relativamente baixa9 .

Em muitos países não existem dados atualizados disponíveis acerca do tabagismo. Os dados são cruciais para a identificação da realidade e das necessidades de avanços e/ou implementações de políticas específicas. Segundo a OMS, o tabaco é um grave problema de saúde pública, pois é o causador de mortes evitáveis nas Américas, matando cerca de 150.000 pessoas na América Latina e na região do Caribe ${ }^{3}$. Ainda sobre a América Latina, a Organização Pan-americana de Saúde (OPAS), destaca a prevalência do uso de tabaco, entre os anos de 1996 a 1999, oscilando entre cerca de $40 \%$, sendo a mais elevada na Argentina e Chile, a 22\%, a mais baixa, no Peru. Em algumas áreas urbanas, mais da metade dos jovens fumam. Entre os homens, a porcentagem dos fumantes varia de 47\%, na Argentina e Chile, a 26\%, na Colômbia. Entre as mulheres a taxa mais alta é de $36 \%$, no Chile, e a mais baixa é de $16 \%$, no Peru. Atualmente não existem dados disponíveis sobre a taxa de fumantes na região do Caribe; relatam, entretanto, que a prevalência varia ente $2 \%$ a $14 \%{ }^{10}$.

O tabagismo se destaca por ser a principal causa prevenível de óbitos nas Américas, matando todos os anos, cerca de 625.000 pessoas, através de doenças crônicas tais como cardiopatias, câncer e doenças pulmonares, contribuindo para que as causas de morte na região passassem das doenças infecciosas para as não infecciosas. $\mathrm{Na}$ América Latina, já há mais pessoas morrendo devido a doenças não infecciosas (muitas destas em decorrência do tabagismo) do que por doenças transmissíveis, afecções maternas e perinatais e carências nutritivas ${ }^{10}$.

\section{Objetivo}

Realizar uma revisão de literatura integrativa, com enfoque nas políticas de saúde de controle ao tabagismo, com o intuito de se analisar o conhecimento produzido na América Latina e Caribe que abordem esta temática.

\section{Método}

Optou-se pela revisão integrativa da literatura como método de pesquisa, pois permite que pesquisas anteriores sejam sumarizadas e conclusões estabelecidas a partir do delineamento das avaliadas, possibilitando a síntese e análise acerca do tema investigado. O propósito inicial para uma revisão integrativa é obter um profundo entendimento de um determinado fenômeno, baseando-se em trabalhos anteriores ${ }^{11}$. É a mais ampla abordagem metodológica referente às revisões, permitindo a inclusão de estudos experimentais e não experimentais para uma compreensão completa do fenômeno analisado. Permite a combinação de dados da literatura teórica e empírica, além de incorporar um vasto leque de propósitos: definição de conceitos, revisão de teorias e evidências, e análise de problemas metodológicos de um tópico particular ${ }^{12}$.

Foram utilizadas as seis etapas propostas por Ganong $^{13}$, que são: identificação do problema ou questionamento; estabelecimento de critérios de inclusão/exclusão de artigos (seleção de amostras); definição de informações a serem extraídas dos artigos selecionados; análise dos dados; interpretação dos resultados; e apresentação da revisão.

Para orientar este estudo, formulou-se a seguinte questão: quais os trabalhos científicos produzidos na literatura latino americana e do Caribe que tratam a temática de políticas de saúde antitabagismo nos países Latino Americanos e Caribe. Na busca dos artigos utilizou-se a seguinte combinação de descritores na língua portuguesa e inglesa: "Política de saúde" e "Tabagismo".

O levantamento bibliográfico foi realizado pela Internet, na BVS (Biblioteca Virtual da Saúde) da Bireme (Biblioteca Regional de Medicina), o acesso gratuito foi realizado no endereço http://www.bvs.br.

Para seleção dos artigos incluídos nesta revisão, utilizou-se a base de dados Lilacs (Literatura Latino-Americana e do Caribe em Ciências da Saúde).

Os critérios de inclusão para seleção dos artigos foram: artigos publicados por países pertencentes à América Latina e Caribe que constam na BVS; artigos indexados nas bases de dados Lilacs. Da amostra total, foram excluídos artigos pelos seguintes critérios: publicações de outros países não pertencentes a América Latina e Caribe indexados no Lilacs; publicações que não retratavam o tema do estudo; textos indexados na base de dados, que não se tratavam de artigos 
científicos; artigos que não foram encontrados na integra no meio digital.

O levantamento dos artigos nas bases de dados Lilacs foi realizado no mês de outubro de 2011, sendo encontrados 50 artigos na busca. Destes 50 artigos, foram excluídas 4 publicações de outros países que não pertenciam à América Latina e Caribe. Procedeu-se, então, a leitura detalhada dos artigos identificando-se 17 que não se enquadravam na temática; 8 textos que não eram artigos; e 5 que não foram encontrados na íntegra em meio digital, restando, portanto, 16, que compuseram a amostra final deste estudo (Tabela 1). Cada artigo selecionado recebeu um código. Este código foi composto pelas letras AC, seguido do número com que o artigo foi identificado na busca efetuada (exemplo: AC 01, onde AC significa Artigo Científico e 01 é o número do artigo encontrado na busca). Esta codificação teve como objetivo facilitar a identificação dos artigos, coleta e análise dos dados.

Para coleta de dados foi elaborado um formulário, contendo os seguintes termos: número da busca; código do estudo; título do artigo; nome do periódico; país de publicação; ano de publicação; autores; objetivos do estudo. Também dos estudos buscou-se vislumbrar os seguintes referenciais teóricos: o estudo é de epidemiologia descritiva e/ou analítica? Sugere políticas? Trata de políticas especificamente de saúde e/ou intersetoriais? Aborda medidas políticas específicas (políticas, programas, projetos), quais? Discute e/ou avalia seus efeitos? Quais efeitos e conclusões?

\section{Resultados}

Como resultado final desta revisão obteve-se 16 artigos científicos, selecionados pelos critérios de inclusão previamente estabelecidos, encontrados na base de dados Lilacs. O Quadro 1 representa as codificações dos artigos e as especificações de cada um dos introduzidos no estudo.

Os 16 artigos selecionados foram comparados e agrupados por similaridade de conteúdos, sob forma de categorias empíricas, sendo divididos em 4 grupos de análise: "espaços livres de fumo", "convenção quadro", "políticas locais brasileiras", e aqueles que não se enquadraram em nenhum desses grupos foram classificados como "outros" (Figura 1).

Destes artigos selecionados, 15 (93,8\%) abordaram estudos epidemiológicos descritivos, apenas $1(6,2 \%)$ o AC 06 trata de abordagem analítica. Todos os artigos de uma forma ou de outra sugerem políticas, projetos ou programas no âmbito do controle do tabagismo (Figura 2).

A seguir apresentamos os grupos que compuseram este estudo.

\section{Espaços livres de fumo}

Cinco artigos (31,2\%) AC $05^{14}, \mathrm{AC} 06^{15}, \mathrm{AC}$ $07^{16}, \mathrm{AC} 08^{17}, \mathrm{AC} 21^{18}$, fizeram parte desta amostra. Os espaços livres de fumo são uma medida de saúde pública recomendada pela OMS, reconhecendo os malefícios causados à saúde dos não fumantes pela exposição à fumaça, que é um cancerígeno tipo A, causador de câncer em humanos. A OMS aponta que a única medida efetiva de saúde pública para evitar a contaminação por esse cancerígeno é a proibição total do fumo em recintos fechados de uso coletivo, públicos ou privados ${ }^{19}$.

\section{Convenção quadro}

Nesse enquadramento 4 artigos (25\%) AC $11^{20}, \mathrm{AC} 17^{21}, \mathrm{AC} 18^{22}, \mathrm{AC} 30^{23}$ fizeram parte. $\mathrm{Na}$ tentativa de controlar o tabagismo em todo o mundo, a OMS coordena um tratado internacional multilateral denominado de "Convenção Quadro para o Controle do Tabagismo", que elaborou um programa para auxiliar os países na redução do fornecimento e do consumo de taba$\mathrm{co}^{3}$. É baseado em seis diretrizes, que receberam o nome MPOWER: M - monitorar o consumo do tabaco e as políticas de prevenção do tabagismo; $\mathrm{P}$ - proteger as pessoas do tabagismo secundário; $\mathrm{O}$ - oferecer ajuda para auxiliar no

Tabela 1. Publicações excluídas.

\begin{tabular}{llr}
\hline \multicolumn{1}{c}{ Base de dados } & \multicolumn{1}{c}{ Critérios de exclusão } & No \\
\hline LILACS & Publicações de outros países que não pertenciam a América Latina e Caribe & 04 \\
& Não se enquadravam na temática & 17 \\
& Textos que não eram artigos & 08 \\
& Artigos que não foram encontrados na íntegra em meio digital & 05 \\
\hline
\end{tabular}


abandono do tabagismo; W (warn) - informar as populações sobre os perigos do tabagismo; E (enforce) - reforçar a proibição de propaganda e campanhas de marketing favoráveis ao tabagismo; R (raises) - aumentar os impostos sobre os produtos do tabaco ${ }^{3}$.

Quadro 1. Artigos levantados na base de dados LILACS sobre políticas de saúde para o controle do tabagismo na América Latina e Caribe.

\begin{tabular}{|c|c|c|c|c|}
\hline $\begin{array}{l}\text { Cód. } \\
\text { Artigo }\end{array}$ & $\begin{array}{c}\text { Título original do } \\
\text { artigo }\end{array}$ & Autores & $\begin{array}{c}\text { Periódico } \\
\left(\text { vol, } \mathbf{n}^{\circ}, \text { pag, ano }\right)\end{array}$ & Considerações/Temática \\
\hline AC 05 & $\begin{array}{l}\text { A resposta da indústria } \\
\text { do tabaco à criação de } \\
\text { espaços livres de fumo } \\
\text { no Brasil }\end{array}$ & $\begin{array}{l}\text { Bialous SA, } \\
\text { Presman S, } \\
\text { Gigliotti A, } \\
\text { Muggli M, Hurt } \\
\text { R }\end{array}$ & $\begin{array}{l}\text { Rev Panam Salud } \\
\text { Publica } \\
\text { 27(4):283-290, } \\
\text { abril } 2010\end{array}$ & $\begin{array}{l}\text { Documenta a reação da } \\
\text { indústria do cigarro à } \\
\text { regulamentação do fumo em } \\
\text { locais públicos no Brasil, } \\
\text { iniciada com legislação em } \\
1996\end{array}$ \\
\hline AC 06 & $\begin{array}{l}\text { Mercadotecnia social y } \\
\text { políticas públicas de } \\
\text { salud: campaña para } \\
\text { promover espacios } \\
\text { libres de humo de } \\
\text { tabaco en México }\end{array}$ & $\begin{array}{l}\text { Villalobos V, } \\
\text { Ramírez OO, } \\
\text { Thrasher JF, } \\
\text { Santillán EA, } \\
\text { Hernández RP, } \\
\text { Cedillo C, } \\
\text { González W }\end{array}$ & $\begin{array}{l}\text { Salud Pública } \\
\text { Méx 52(2):129- } \\
\text { 137, } 2010\end{array}$ & $\begin{array}{l}\text { Descreve campanha massiva, } \\
\text { promovida por meio de } \\
\text { mídias eletrônicas, para } \\
\text { promover espaços livres de } \\
\text { fumo }\end{array}$ \\
\hline AC 07 & $\begin{array}{l}\text { Informing effective } \\
\text { smokefree policies in } \\
\text { Argentina: air quality } \\
\text { monitoring study in } \\
15 \text { cities (2007-2009) }\end{array}$ & $\begin{array}{l}\text { Schoj V, Sebrié } \\
\text { EM, Pizarro } \\
\text { ME, Hyland A, } \\
\text { Travers MJ }\end{array}$ & $\begin{array}{l}\text { Salud Pública } \\
\text { Méx. } 52 \text { (Supl. } \\
\text { 2):157-67, } 2010\end{array}$ & $\begin{array}{l}\text { Avaliar a contaminação, pela } \\
\text { fumaça do cigarro, no setor } \\
\text { gastronômico da Argentina, } \\
\text { através da medição dos níveis } \\
\text { de partículas respiráveis }\end{array}$ \\
\hline AC 08 & $\begin{array}{l}\text { Exposición a humo de } \\
\text { tabaco ajeno en } \\
\text { lugares públicos de la } \\
\text { Ciudad de México, } \\
\text { Guadalajara y } \\
\text { Monterrey }\end{array}$ & $\begin{array}{l}\text { Reynales- } \\
\text { Shigematsu LM, } \\
\text { Ortega-Ceballos } \\
\text { PA, Gimeno D, } \\
\text { Barrientos- } \\
\text { Gutierrez T }\end{array}$ & $\begin{array}{l}\text { Salud pública } \\
\text { Méx. } 52 \text { (Supl. } \\
\text { 2):168-171, } 2010\end{array}$ & $\begin{array}{l}\text { Quantificar a exposição à } \\
\text { fumaça do tabaco em lugares } \\
\text { públicos no México, a fim } \\
\text { de impulsionar ambientes } \\
\text { políticos locais } 100 \% \text { livres } \\
\text { de fumo }\end{array}$ \\
\hline AC 09 & $\begin{array}{l}\text { Políticas de género de } \\
\text { ámbito global en el } \\
\text { control y la } \\
\text { prevención del } \\
\text { tabaquismo }\end{array}$ & Nerin I, Jane M & $\begin{array}{l}\text { Salud pública } \\
\text { Méx. } 52 \text { (Supl. } \\
\text { 2):304-314, } 2010\end{array}$ & $\begin{array}{l}\text { Enfoca a crescente } \\
\text { prevalência de tabagismo } \\
\text { entre as mulheres, } \\
\text { levantando questões de } \\
\text { gênero na concepção de } \\
\text { políticas de prevenção e } \\
\text { tratamento do tabagismo. }\end{array}$ \\
\hline AC 11 & $\begin{array}{l}\text { La etapa de los bienes } \\
\text { globales en salud, una } \\
\text { perspectiva nacional }\end{array}$ & $\begin{array}{l}\text { Córdova- } \\
\text { Villalobos JA }\end{array}$ & $\begin{array}{l}\text { Salud pública } \\
\text { Méx. 50(S3):309- } \\
\text { 14, } 2008\end{array}$ & $\begin{array}{l}\text { Trata da Convenção-Quadro } \\
\text { para o Controle do } \\
\text { Tabagismo, levando à criação } \\
\text { da Lei Geral para o Controle } \\
\text { do tabagismo, um grande } \\
\text { marco na política sanitária } \\
\text { mexicana, visando a } \\
\text { contenção da epidemia do } \\
\text { tabaco no país }\end{array}$ \\
\hline AC 12 & $\begin{array}{l}\text { Prevenção às drogas } \\
\text { nas escolas: uma } \\
\text { experiência pensada a } \\
\text { partir dos modelos de } \\
\text { atenção em saúde }\end{array}$ & $\begin{array}{l}\text { Müller AC, Paul } \\
\text { CL, Santos NIS }\end{array}$ & $\begin{array}{l}\text { Estud. Psicol. } \\
\text { 25(4):607-616, } \\
\text { out/dez } 2008\end{array}$ & $\begin{array}{l}\text { Analisa um projeto de } \\
\text { prevenção às drogas no } \\
\text { ambiente escolar e discute a } \\
\text { relação desta proposta com } \\
\text { os modelos de atenção em } \\
\text { saúde }\end{array}$ \\
\hline
\end{tabular}




\section{Políticas locais brasileiras}

Quanto às "políticas locais brasileiras", 03 artigos $(18,8 \%)$ compuseram esta amostra AC
$24^{24}$, AC $25^{25}$, AC $26^{26}$. O Brasil possui um Programa Nacional para o Controle do Tabagismo, onde destacam-se ações referentes à proibição da publicidade, propaganda e advertências im-

Quadro 1. continuação

\begin{tabular}{|c|c|c|c|c|}
\hline $\begin{array}{l}\text { Cód. } \\
\text { Artigo }\end{array}$ & $\begin{array}{c}\text { Título original do } \\
\text { artigo }\end{array}$ & Autores & $\begin{array}{c}\text { Periódico } \\
(\text { vol, no, pag, ano })\end{array}$ & Considerações/Temática \\
\hline AC 13 & $\begin{array}{l}\text { The role of public } \\
\text { policies in reducing } \\
\text { smoking prevalence } \\
\text { and deaths: the } \\
\text { Argentina Tobacco } \\
\text { Policy Simulation } \\
\text { Model }\end{array}$ & $\begin{array}{l}\text { Ferrante D, Levy } \\
\text { D, Peruga A, } \\
\text { Compton C, } \\
\text { Romano E }\end{array}$ & $\begin{array}{l}\text { Rev Panam Salud } \\
\text { publica 21(1):37- } \\
49,2007\end{array}$ & $\begin{array}{l}\text { Comparou políticas de } \\
\text { controle ao tabagismo de } \\
\text { forma independente e como } \\
\text { um todo por um modelo de } \\
\text { simulação para projetar a } \\
\text { prevalência do tabagismo e } \\
\text { da mortalidade associada a } \\
\text { esse hábito na Argentina } \\
\text { desde } 2001\end{array}$ \\
\hline AC 17 & $\begin{array}{l}\text { Evaluación de las } \\
\text { políticas contra el } \\
\text { tabaquismo en países } \\
\text { latinoamericanos en la } \\
\text { era del Convenio } \\
\text { Marco para el Control } \\
\text { del Tabaco }\end{array}$ & $\begin{array}{l}\text { Thrasher JF, } \\
\text { Chaloupka F, } \\
\text { Hammond D, } \\
\text { Fong G, Borland } \\
\text { R, Hastings G, } \\
\text { Cummings M }\end{array}$ & $\begin{array}{l}\text { Salud pública } \\
\text { Méx } \\
48(\text { Supl.1):155- } \\
166,2006\end{array}$ & $\begin{array}{l}\text { Aborda o papel da } \\
\text { Convenção-Quadro para } \\
\text { Controle do Tabagismo, no } \\
\text { intuito de coordenar } \\
\text { políticas globais que reduzam } \\
\text { o consumo do tabaco, e } \\
\text { quais impactos terão estas } \\
\text { medidas na renda média e } \\
\text { baixa }\end{array}$ \\
\hline AC 18 & $\begin{array}{l}\text { El argumento de } \\
\text { responsabilidad social } \\
\text { de la industria } \\
\text { tabacalera en Brasil }\end{array}$ & $\begin{array}{l}\text { Cavalcante T, } \\
\text { Carvalho AM, } \\
\text { Rangel EC }\end{array}$ & $\begin{array}{l}\text { Salud pública } \\
\text { Méx 48(Supl.1): } \\
\text { 173-182, } 2006\end{array}$ & $\begin{array}{l}\text { Expõe a questão da } \\
\text { responsabilidade social } \\
\text { corporativa, e as várias } \\
\text { experiências positivas do } \\
\text { Brasil na abordagem das } \\
\text { estratégias da indústria do } \\
\text { tabaco para minar os } \\
\text { esforços nacionais de } \\
\text { controle do tabaco }\end{array}$ \\
\hline AC 19 & $\begin{array}{l}\text { Política fiscal y control } \\
\text { del tabaco: una } \\
\text { oportunidad única } \\
\text { para beneficiar a la } \\
\text { salud pública y al } \\
\text { erario }\end{array}$ & $\begin{array}{l}\text { Armendares PE, } \\
\text { Reynales } \\
\text { Shigematsu LM }\end{array}$ & $\begin{array}{l}\text { Salud pública } \\
\text { Méx 48(Supl.1): } \\
\text { 167-172, } 2006\end{array}$ & $\begin{array}{l}\text { Aborda o aumento do preço } \\
\text { do tabaco através de } \\
\text { impostos como sendo uma } \\
\text { das ferramentas mais } \\
\text { eficientes em uma política } \\
\text { global para combater o } \\
\text { tabagismo }\end{array}$ \\
\hline AC 21 & $\begin{array}{l}\text { Espacios } 100 \text { por } \\
\text { ciento libres de humo: } \\
\text { una realidad en el } \\
\text { Distrito Federal }\end{array}$ & $\begin{array}{l}\text { Guillermo- } \\
\text { Tenorio X }\end{array}$ & $\begin{array}{l}\text { Salud pública } \\
\text { Méx. } 50 \\
\text { (Supl.3):384-390, } \\
2008\end{array}$ & $\begin{array}{l}\text { Descreve o processo } \\
\text { legislativo das leis que } \\
\text { criaram os espaços } 100 \% \\
\text { livres do fumo }\end{array}$ \\
\hline AC 24 & $\begin{array}{l}\text { Experiencia brasileña } \\
\text { con políticas de } \\
\text { control del } \\
\text { tabaquismo }\end{array}$ & Cavalcante $\mathrm{T}$ & $\begin{array}{l}\text { Salud pública } \\
\text { Méx. 46(6):549- } \\
558,2004\end{array}$ & $\begin{array}{l}\text { Trata da articulação de } \\
\text { estratégias e esforços de } \\
\text { diferentes grupos sociais, } \\
\text { governamentais e não } \\
\text { governamentais, a partir de } \\
\text { uma óptica de promoção da } \\
\text { saúde para controlar o uso } \\
\text { do fumo }\end{array}$ \\
\hline
\end{tabular}


Quadro 1. continuação

\begin{tabular}{|c|c|c|c|c|}
\hline $\begin{array}{l}\text { Cód. } \\
\text { Artigo }\end{array}$ & $\begin{array}{c}\text { Título original do } \\
\text { artigo }\end{array}$ & Autores & $\begin{array}{c}\text { Periódico } \\
\left(\text { vol, } \mathbf{n}^{\mathbf{o}}, \text { pag, ano }\right)\end{array}$ & Considerações/Temática \\
\hline AC 25 & $\begin{array}{l}\text { O Instituto Nacional } \\
\text { do Câncer e o } \\
\text { controle do } \\
\text { tabagismo: uma } \\
\text { análise da gestão } \\
\text { federal do tratamento } \\
\text { do tabagismo no SUS }\end{array}$ & Carvalho CRS & Fiocruz, 2009 & $\begin{array}{l}\text { Caracterizar o modelo atual } \\
\text { de gestão do tratamento do } \\
\text { tabagismo pelo INCA e } \\
\text { analisar, na gestão federal, } \\
\text { estadual e municipal, os } \\
\text { problemas e pontos de } \\
\text { estrangulamentos da } \\
\text { estratégia do tratamento do } \\
\text { tabagismo na rede SUS }\end{array}$ \\
\hline AC 26 & $\begin{array}{l}\text { Política de controle do } \\
\text { tabagismo em Recife } \\
\text { (PE): fortalecendo o } \\
\text { modelo de gestão com } \\
\text { enfoque na promoção } \\
\text { à saúde }\end{array}$ & $\begin{array}{l}\text { Menezes MP, } \\
\text { Campos Neta TJ }\end{array}$ & $\begin{array}{l}\text { Divulg. saúde } \\
\text { debate. } \\
\text { (42):68-79, abr } \\
2008\end{array}$ & $\begin{array}{l}\text { Sistematizou a política de } \\
\text { Controle do Tabagismo e } \\
\text { Outros Fatores de Risco de } \\
\text { Câncer da Secretaria } \\
\text { Municipal de Saúde do } \\
\text { Recife, em desenvolvimento } \\
\text { no período de gestão 2001- } \\
\text { 2007, incluindo os desafios } \\
\text { para 2008. Com destaque } \\
\text { para os projetos inclusos no } \\
\text { Programa Ambiente Livre de } \\
\text { Fumo }\end{array}$ \\
\hline AC 30 & $\begin{array}{l}\text { Política de controle do } \\
\text { tabagismo no Distrito } \\
\text { Federal }\end{array}$ & $\begin{array}{l}\text { Mauchi SDN, } \\
\text { Silva CAR }\end{array}$ & $\begin{array}{l}\text { Brasília méd. } \\
\text { 36(3/4):106-109, } \\
1999\end{array}$ & $\begin{array}{l}\text { Discorre sobre a atuação da } \\
\text { Secretaria de Saúde do } \\
\text { Distrito Federal no } \\
\text { desenvolvimento de uma } \\
\text { política de Controle do } \\
\text { tabagismo, com base nos } \\
\text { programas nacionais } \\
\text { preconizados pelo Instituto } \\
\text { Nacional do Câncer }\end{array}$ \\
\hline
\end{tabular}

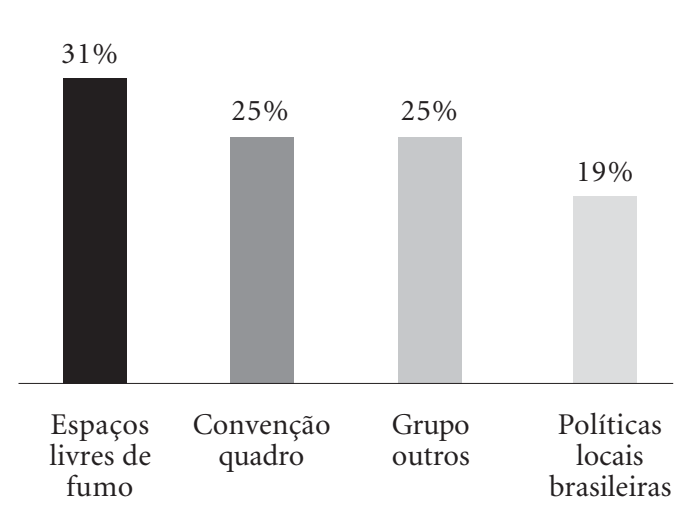

Figura 1. Divisão empírica por grupo de estudo.

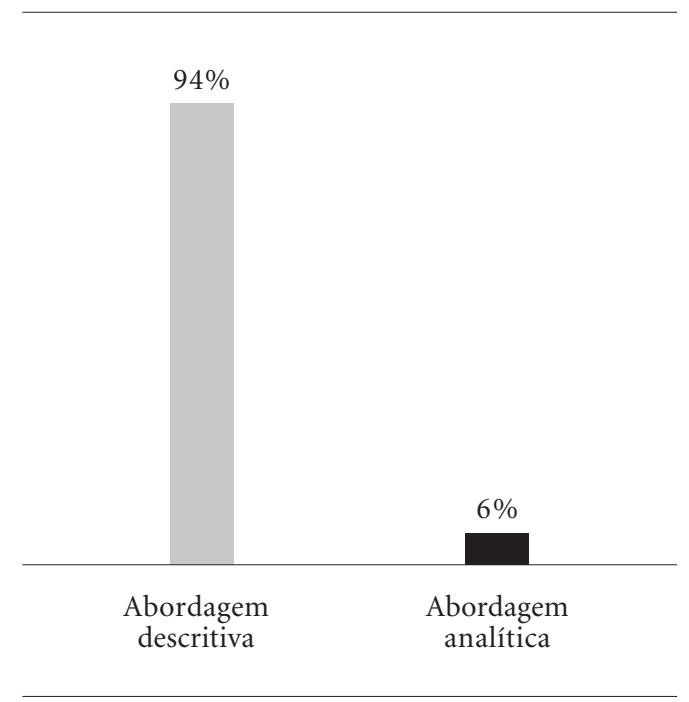

Figura 2. Classificação do estudo por abordagem, se descritiva ou analítica. 
pressas nas embalagens de produtos derivados do tabaco. O Brasil foi o primeiro país a proibir adjetivos enganosos nos maços de cigarros, como light ou suave, porém, o foco das ações ainda está concentrado nas ações não relacionadas aos $\operatorname{preços}^{27}$.

\section{Grupo outros}

O grupo "outros" conta 4 artigos (25\%) AC $09^{28}, \mathrm{AC} 12^{29}, \mathrm{AC} 13^{30}, \mathrm{AC} 19^{31}$ que retratam políticas diversas voltadas ao controle do tabagismo.

\section{Discussão}

Os artigos do grupo "espaços livres de fumo" abordam políticas de saúde com abrangências intersetoriais para sua implementação, bem como, suas dificuldades nesse processo nos países da América Latina e Caribe. Discutem e avaliam a criação dos processos legislativos para a implementação e regulamentação da criação de espaços livres da exposição à fumaça do cigarro, considerando os direitos à saúde dos não fumantes. Os efeitos destas políticas baseiam-se nas inadequações e desacordos com a Convenção Quadro, em função do forte interesse das indústrias do cigarro, embora também haja algumas implementações positivas. Assinalam também para outras perspectivas de sucessos de políticas já implementadas por meio de marketing sociais, monitoramento e avaliação de medidas de poluição ambiental em reposta às políticas de controle do tabagismo, mostrando baixos níveis de poluição nos locais onde esta política se encontra vigente. O sucesso da implementação dessas políticas, nesses países, demonstram a soberania do poder legislativo, por meio da lei, que fortalece o Estado de direito às ações concretas e garantem o respeito constitucional do Estado em benefício da sociedade.

Os artigos "convenção quadro" sugerem políticas intersetoriais como: desenvolvimento sustentável, financeiro, educacional, social, de recursos humanos, agricultura, etc. Discutem a importância de impulsionar políticas globais, segundo a Convenção Quadro, políticas e programas de saúde que visam ações de controle do tabagismo, assim como, avaliação dos efeitos da implantação destas políticas em países de renda menor do que aquelas em que a Convenção Quadro foi inicialmente proposta. Demonstram alguns dos esforços do Brasil na luta contra as estratégias das indústrias tabaqueiras, que utili- zam o desenvolvimento sustentável e a responsabilidade social numa tentativa de criar imagens positivas, visando desviar a atenção dos danos sociais de seus produtos. Por fim, procuram dar aos Estados Membros, os recursos necessários para controlar a pandemia, mobilizando fundos e recursos humanos adequados para garantir a continuidade do programa (Convenção Quadro) e fortalecer a colaboração com as Organizações não Governamentais. O objetivo é proteger as gerações presentes e futuras das devastadoras consequências sanitárias, a exposição social, econômica e ambiental do tabaco.

No que tange a "políticas locais brasileiras" o governo já atende a muitas das provisões da Convenção-Quadro para o Controle do Tabaco (CQCT), assinada em 2003 e ratificada em 2005. O Programa inclui vigilância, legislação e incentivos econômicos para a diversificação de culturas, além de educação em escolas, locais de trabalho e nas unidades de saúde. Como passos necessários, o programa identifica: prevenir a iniciação e experimentação do tabaco entre as crianças e adolescentes; promover ações para estimular a cessação; proteger os não fumantes dos perigos da fumaça ambiental do tabaco; e promover a redução dos danos causados pelo tabaco, através de medidas de regulamentação do produto ${ }^{27}$.

Dois dos artigos AC 24 e AC 26 abordam políticas exclusivas de saúde, e um (AC 25), aborda estratégias governamentais abrangentes, das especificidades da política nacional de saúde em suas dimensões de proteção social, econômica e de poder. Os artigos discutem, no âmbito nacional, a preparação de uma base de apoio para atender as diversas obrigações decorrentes da Convenção Quadro de Controle ao Tabagismo, identificam como problema a limitada oferta de tratamento do tabagismo no Sistema Único de Saúde e as dificuldades de gestão da estratégia nas três esferas de governo. Destacam também os projetos inclusos no Ambiente Livre de Fumo, implantados e acompanhados pela coordenação de "Controle de Tabagismo e Outros Fatores de Risco para o Câncer". Como efeitos da implementação das políticas, além de se preparar uma maior consolidação de apoio nacional, busca-se proposições voltadas para o enfrentamento de algumas causas dos problemas identificados, relacionados à articulação inter e intragovernamental, ao planejamento e à coordenação das ações logísticas e assistenciais na rede de serviço do sistema nacional de saúde. Apresenta ainda como resultado, o exemplo da cidade de Recife (Pernambuco), onde as ações de promoção, preven- 
ção e assistência contribuíram para a diminuição da prevalência de fumantes na população de $28 \%$ para 14,7\%. Um estudo realizado em 2008 aponta para o fato da exposição passiva ao tabagismo, que no Brasil é de cerca de 33\%, sendo considerada elevada, visto que expõe os fumantes passivos aos mesmos malefícios aos quais estão expostos os fumantes ativos ${ }^{32}$. No "grupo outros” AC 09 refere-se à política de gênero, de âmbito global e intersetorial, sugerindo políticas voltadas para as questões próprias do tabagismo na mulher, de acordo com suas especificidades, com foco na proteção, prevenção e tratamento. Procura incorporar a perspectiva de gênero na elaboração das políticas públicas. Avalia os efeitos da implementação de políticas de saúde para a prevenção e a cessação do tabagismo em mulheres, considerando estratégias e linhas políticas que contemplem aspectos legislativos específicos ao gênero, como medidas educacionais e assistenciais, assim como medidas de investigação e avaliação dos programas. O AC12 diz respeito à análise de um projeto intersetorial de prevenção às drogas, no ambiente escolar, realizado em uma escola, no município de Santa Maria (RS) e discute a relação dessa proposta com os modelos de atenção à saúde. Sugere políticas de prevenção do uso de drogas na escola, incluindo o tabagismo. Discute a participação dos sujeitos sociais no processo de produção da saúde, como um modelo em Defesa da Vida. Visa contribuir para a problematização de escolhas metodológicas e seus efeitos em abordagens de prevenção às drogas na escola. A experimentação do tabaco entre estudantes é um problema importante, estando associado à adição ao tabagismo, visto que metade dos adolescentes que experimentaram o cigarro correm o risco de se tornarem fumantes ${ }^{33,34}$.

O AC 13 visa a comparar as políticas de controle do tabaco, de forma independente, e na forma de um conjunto de medidas, por meio de um projeto denominado Modelo de Simulação para a prevalência do tabagismo e projeção da taxa de mortalidade prematura. Sugere políticas intersetoriais, abrangentes e descreve um modelo de planejamento preliminar (Modelo de Simulação da Política do Tabaco). Estima taxas de tabagismo futuro e mortes atribuíveis ao tabagismo segundo idade e sexo, bem como avalia o efeito das políticas de controle, identificando os dados necessários para a supervisão das consequências e avaliações políticas necessárias. Como efeito demonstra uma combinação de políticas que envolvam o aumento de $80 \%$ dos impostos sobre o preço do cigarro, ambientes livres de tabaco, aplicação de publicidade, fiscalização e proibição total de publicidade em propagandas de cigarro, bem como advertências sanitárias, e controle rigoroso de acesso aos jovens ao cigarro. E o AC 19 trata de uma política de ação intersetorial, focando como os impostos contribuem para promover a cessação, reduzindo o consumo e o número de mortes atribuíveis ao tabagismo. Discute as possibilidades na obtenção desses benefícios de saúde associados com aumentos de impostos, sem consequências econômicas negativas, como o desemprego e prejuízos fiscais. Como efeito discute como é necessário enfrentar a poderosa indústria do tabaco, que conhecem a eficiência fiscal para combater o tabagismo e utilizam campanhas de propaganda intensa, lobby político e negociação de acordos voluntários para regularem a si mesmos, a fim de evitar constrição legal ou fiscal.

\section{Conclusão}

A epidemia do tabagismo é um problema de saúde pública globalizado que transcende fronteiras de países. As medidas intersetoriais podem comprovadamente ser efetivas para controlar sua expansão, sendo a eficácia dessas medidas dependentes de uma ampla cooperação internacional, como a que tem sido proposta pela OMS, através da Convenção Quadro de Controle ao Tabagismo.

Experiências positivas vêm sendo destacadas pelos países da América Latina e Caribe na luta contra as estratégias da indústria do tabaco, demonstrando o sucesso desta rede conjunta, a fim de desempenhar um papel fundamental no monitoramento e fiscalização de políticas públicas para o controle de tabagismo. Neste sentido, a Convenção-Quadro representa a essência de uma extensa rede em escala internacional, que decorre da convicção de que o controle global do consumo de tabaco depende de esforços conjuntos transnacionais, tanto governamentais como não governamentais para proteger as gerações presentes e futuras das devastadoras consequências geradas pelo consumo e exposição ao tabaco. Com isso pode-se dizer que a Convenção-Quadro é um compromisso para proteger as populações da interferência indevida da indústria do tabaco. Isto leva a um grande desafio, especialmente para os países que ratificaram a Convenção Quadro, sobre como manter uma interface com a indústria do tabaco, sem permitir a inter- 
ferência indevida desta nas políticas de controle do tabaco nestes países.

Uma das observações, que cabe ser ressaltada após análises dos estudos, é que poucos dados sobre o tabagismo na América Latina e Caribe estão disponíveis, principalmente no que tange a avaliação das políticas implantadas, bem como dados sobre a epidemiologia do tabagismo nos países desta região. Destaques foram dados principalmente às políticas de espaço livres de fumo (5 artigos) e que abordem alguns aspectos da Convenção Quadro de Controle do Tabagismo (4 artigos), além dos que tratavam de políticas brasileiras de enfretamento ao tabagismo (3 artigos).

\section{Colaboradores}

LGS Fagundes participou na concepção, metodologia, pesquisa, elaboração do texto e revisão; MG Martins participou na concepção, pesquisa, elaboração e revisão; PCR Palmiéri participou na pesquisa; e SI Silva Júnior participou na concepção do estudo. EMS Magalhães participou na concepção, pesquisa e elaboração do texto.
Os países pertencentes à região da América Latina e Caribe vêm concentrando esforços para combater os produtos do cigarro. No entanto, o que se percebe ainda, é que existe um tom de adversidade entre a indústria tabaqueira e aqueles que se opõem a ela. Esta indústria tenta minar os esforços desses países para se manterem líderes de mercado e gerar mais lucro, sem se importar com os danos sociais, ambientais, econômicos e na saúde que causam. Cabe a todos os países da região da América Latina e Caribe não se sujeitarem a pressão impostas por esta indústria, coordenando ações que são destacadas pela Convenção Quadro de Controle ao tabagismo, através do MPOWER, de acordo com a realidade de cada país. 


\section{Referências}

1. Silveira AF. Tabagismo e politicas públicas: uma análise sobre a lógica de diferentes estabelecimentos do ramo de entretenimento sobre a proibição de fumar em ambientes fechados [tese]. São Paulo: Pontifícia Universidade Católica; 2007.

2. Sutherland G. Smoking: can we really make a difference? Heart. 2003; 89(Supl. 2):ii25-ii27.

3. World Health Organization (WHO). Report on the Global tobacco epidemic, 2008: The MPOWER package. Geneva: WHO; 2008.

4. Lopez AD, Mathers CD, Ezzati M, Jamison DT, Murray CJ. Global and regional burden of disease and risk factors, 2001: systematic analysis of population health data. Lancet 2006; 367(9524):1747-1757.

5. Doll R, Peto R, Borehan J, Sutherland I. Mortality in relation to smoking: 50 years' observations on male british doctors. Br J Cancer 2004; 92(3):426-429.

6. Brasil. Ministério da Saúde (MS). Secretaria de Políticas de Saúde Departamento de Formulação de Políticas de Saúde. Políticas de saúde metodologia de formulação. Brasília: MS; 1998.

7. Silva IF. O processo decisório nas instâncias colegiadas do SUS no estado do Rio de Janeiro [dissertação]. Rio de Janeiro: Fiocruz; 2000.

8. Monteiro CA, Cavalcante TM, Moura EC, Claro RM, Szwarcwald CL. Population - based evidence of a strong decline in the prevalence of smokers in Brazil (1989-2003). Bull World Health Org 2007; 85(7):527-534.

9. Lopez A, Collishaw N, Piha T. A descriptive model of the cigarette epidemic in developed countries. Tobacco Control 1994; 3(3):242-247.

10. Organização Pan-Americana de Saúde (OPAS). Tabaco nas Américas: 31 de maio de 2000 - Dia Mundial sem Tabaco. Brasília: OPAS; 2000. Informativo.

11. Broome ME. Integrative literature reviews for the development of concepts. In: Rodgers BL, Knafl KA. Concept development in nursing: foundations, techniques and applications. Philadelphia: Saunders; 2000. p. 231-250.

12. Whittemore R, Knafl $\mathrm{K}$. The integrative review: update methodology. J Adv Nurs 2005; 52(5):546553.

13. Ganong LH. Integrative Reviews of nursing research. Res Nurs health 1987; 10(1):1-11.

14. Bialous SA, Presman S, Gigliotti A, Muggli M, Hurt R. A resposta da indústria do tabaco à criação de espaços livres de fumo no Brasil. Rev Panam Salud Publica 2010; 27(4):283-290.

15. Villalobos V, Ramírez OO, Thrasher JF, Santillán EA, Hernández RP, Cedillo C, González W. Mercadotecnia social y políticas públicas de salud: campaña para promover espacios libres de humo de tabaco en México. Salud Pública Méx 2010; 52(2):129137.

16. Schoj V, Sebrié EM, Pizarro ME, Hyland A, Travers MJ. Informing effective smokefree policies in Argentina: air quality monitoring study in 15 cities (2007-2009). Salud Pública Méx 2010; 52(Supl. 2):157167.
17. Reynales-Shigematsu LM, Ortega-Ceballos PA, Gimeno D, Barrientos-Gutierrez T. Exposición a humo de tabaco ajeno en lugares públicos de la Ciudad de México, Guadalajara y Monterrey. Salud pública Méx 2010; 52(Supl. 2):S168-S171.

18. Guillermo-Tenorio X. Espacios $100 \%$ libres de humo: una realidad en el Distrito Federal. Salud pública Méx 2008; 50(Supl. 3):S384-S390.

19. World Health Organization (WHO). Protection from exposure to second-hand tobacco smoke. Policy recommendations. Genebra: WHO; 2007. Policy recommendations.

20. Cordova-Villalobos JA. La etapa de los bienes globales en salud, una perspectiva nacional. Salud pública Méx. 2008; 50(Supl. 3):309-314.

21. Thrasher JF, Chaloupka F, Hammond D, Fong G, Borland R, Hastings G, Cummings M. Evaluación de las políticas contra el tabaquismo en países latinoamericanos en la era del Convenio Marco para el Control del Tabaco. Salud pública Méx 2006; 48(Supl. 1):S155-S166.

22. Cavalcante T, Carvalho AM, Rangel EC. El argumento de responsabilidad social de la industria tabacalera en Brasil. Salud pública Méx 2006; 48(Supl. 1):S173-S182.

23. Mauchi SDN, da Silva CAR. Política de controle do tabagismo no Distrito Federal. Brasilia méd_1999; 36(3/4):106-109.

24. Cavalcante T. Experiencia brasileña con políticas de control del tabaquismo. Salud pública de Méx. 2004; 46(6):549-558.

25. Carvalho CRS. O Instituto Nacional do Câncer e o controle do tabagismo: uma análise da gestão federal do tratamento do tabagismo no SUS. Rio de Janeiro: Fiocruz; 2009.

26. Menezes MP, Campos Neta TJ. Política de controle do tabagismo em Recife (PE): fortalecendo o modelo de gestão com enfoque na promoção à saúde. Divulg. saúde debate 2008; (42):68-79.

27. Iglesias $\mathrm{R}$, Jha $\mathrm{P}$, Pinto $\mathrm{M}$, da Costa e Silva VL, Godinho J. Controle de tabagismo no Brasil [Internet]. Washington: The World Bank; 2007 [cited 2011 Nov 10]. 119p. Available from: http://portal.saude. gov.br/portal/arquivos/pdf/ControledoTabagismo noBrasil.pdf

28. Nerin I, Jane M. Políticas de género de ámbito global en el control y la prevención del tabaquismo. Salud pública 2010; 52(Supl. 2):S304-S314.

29. Muller AC, Paul CL, dos Santos NIS. Prevenção às drogas nas escolas: uma experiência pensada a partir dos modelos de atenção em saúde. Estud. psicol. 2008; 25(4):607-616.

30. Ferrante D, Levy D, Peruga A, Compton C, Romano $\mathrm{E}$. The role of public policies in reducing smoking prevalence and deaths: the Argentina Tobacco Policy Simulation Model. Rev Panam Salud Publica 2007; 21(1):37-49. 
31. Armendares PE, Reynales Shigematsu LM. Política fiscal y control del tabaco: una oportunidad única para beneficiar a la salud pública y al erario. Salud pública Méx 2006; 48(Supl. 1):S167-S172.

32. Passos VM, Giatti L, Barreto, AM. Tabagismo passivo no Brasil: resultados da Pesquisa Especial do Tabagismo. Cien Saude Colet 2008; 16(9):3671-3678.

33. Barreto SM, Giatti L, Cado L, Moura L, Crespo C, Malta DC. Exposição ao tabagismo entre escolares no Brasil. Cien Saude Colet 2008 Out; 15(Supl. 2):30273034.

34. Gilpin EA, White VM, Pierce JP. What fraction of young adults are at risk for future smoking, and who are they? Nicotine Tob Res. 2005; 7(5):747-759.

Artigo apresentado em 04/08/2012

Aprovado em 05/10/2012

Versão final apresentada em 18/10/2012 